\title{
TOXICIDADE DE FLÚOR EM CULTIVARES DE MILHO EM ÁREA PRÓXIMA A UMA INDÚSTRIA CERÂMICA, ARARAS (SP) ${ }^{(1)}$
}

\author{
CAIO FORTES $^{(2)}$; AILDSON PEREIRA DUARTE ${ }^{(3)} ;$ SIZUO MATSUOKA $^{(4)}$, \\ HERMANN P. HOFFMANN ${ }^{(4)}$, NORBERTO ANTÔNIO LAVORENTI ${ }^{(5)}$
}

\begin{abstract}
RESUMO
Indústrias que submetem material terroso a altas temperaturas como a de cerâmicas, emitem fluoretos para a atmosfera na forma de gás, particulado ou ácido fluorídrico. Em experimentos de milho (Zea mays L.), do Centro de Ciências Agrárias da Universidade Federal de São Carlos, Araras (SP), as plantas apresentaram sintomas que evoluíam de uma descoloração do limbo foliar até o secamento marginal das folhas. O objetivo deste trabalho foi avaliar os teores foliares de flúor (F) na cultura do milho com sintomas de toxicidade causados por fluoretos atmosféricos emitidos por uma indústria cerâmica vizinha, e possíveis diferenças entre as cultivares de milho quanto à severidade desses sintomas. Em um experimento atribuíram-se notas relativas ao grau de injúria das folhas de 22 cultivares e, em outro, foi feita determinação de $\mathrm{F}$ em três cultivares de milho semeadas a 350 e 1.000 metros de distância daquela indústria. Os teores foliares de $\mathrm{F}$ nas folhas de milho estavam altos nas duas distâncias. Para o milho "safrinha" os teores chegaram até quatro vezes acima do teor crítico, variando entre 126 e $160 \mathrm{mg} . \mathrm{kg}^{-1}$. Constataram-se diferenças entre as cultivares de milho quanto à severidade de sintomas, mas não foi possível estabelecer correlação com danos na produtividade.
\end{abstract}

Palavras-chave: clorose, diagnose visual, análise foliar, produtividade de grãos.

\author{
ABSTRACT \\ FLUORINE TOXICITY IN CORN PLANTS NEARBY A CERAMIC INDUSTRY, ARARAS, \\ STATE OF SÃO PAULO, BRAZIL
}

The emission of fluoride gases, particles or fluoridric acid is commonly observed in industries that submit terrigenous materials to high temperatures, such as in the ceramic industry. Unusual abnormalities in leaves of adult plants that have been suspected to be fluorine toxicity have been observed in corn experiments carried out in the Center of Agricultural Sciences of the Federal University of São Carlos, Araras, SP, Brazil. The symptoms in the corn leaves started as discolouring of internerval cells of completed developed leaves followed by marginal burning of its tips. The objectives of this work

$\left({ }^{1}\right)$ Recebido para publicação em 19 de agosto de 2002 e aceito em 27 de março de 2003.

$\left(^{2}\right)$ Graduando de Engenharia Agronômica da Universidade Federal de São Carlos campus Araras (SP) - Centro de Ciências Agrárias e membro do Grupo de Estudo e Pesquisa em Agricultura (GEPAGRI/CCA/UFSCar). E-mail: caiofortes@bol.com.br

$\left({ }^{3}\right)$ Instituto Agronômico/Pólo Regional de Desenvolvimento Tecnológico dos Agronegócios do Médio Paranapanema, Caixa Postal 263, 19800-000 Assis (SP). E-mail: aildson@femanet.com.br

$\left({ }^{4}\right)$ Departamento de Biotecnologia Vegetal (DBV/CCA/UFSCar), Caixa Postal 253, 13610-970 Araras (SP). E-mail: sizuo@dbv.cca.ufscar.br; hermann@dbv.cca.ufscar.br

$\left({ }^{5}\right)$ Departamento de Tecnologia Agroindustrial e Sócio-Economia Rural (DTAISER/CCA/UFSCar), Araras (SP). Email:norba@cca.ufscar.br 
was to evaluate the amount of fluorine (F) in corn leaves with injury symptoms, probably from airborne fluorides nearby a ceramic industry, and to verify differences among corn cultivars for the symptoms severity. Leaf analysis in samples collected in corn plants growing 350 and $1.000 \mathrm{~m}$ far from a ceramic industry confirmed fluorine concentration as high as four times the normal, ranging from 126 to $160 \mathrm{mg} \mathrm{kg}^{-1}$. Although the genotypes in those two experimental fields showed distinct reactions, it was not possible to correlate any reduction in production with severity of symptoms.

Key words: clorosis, visual diagnosis, leaf analysis, grains yield.

\section{Introdução}

A região que compreende o Pólo Cerâmico de Santa Gertrudes, no Estado de São Paulo, e que abrange o município de Araras, reúne cerca de 40 indústrias cerâmicas, gerando $60 \%$ da produção brasileira de pisos esmaltados (Associação..., 2001). Além da atividade ceramista, a agricultura também se destaca na região, sendo expressivos os cultivos de cana-de-açúcar, citros, eucalipto e culturas anuais como o milho.

Indústrias que submetem rochas e outros tipos de material terroso a altas temperaturas, como a de cerâmicas, vidros, fertilizantes, fundições e siderúrgicas emitem fluoretos para a atmosfera na forma de gás, particulado, ou ácido fluorídrico (HF). O excesso de flúor (F) e de outros poluentes atmosféricos pode acarretar sérios danos à vegetação (AzEvedo et al., 1993; ArNDT et al., 1995; FialHo, 1997; Figueiredo, 1994; LoPEs et al., 1998). A CETESB, ao avaliar o estágio de degradação do ambiente no município de Cordeirópolis (SP), integrante do citado pólo, constatou contaminação por fluoretos em plantas indicadoras de Dracaena alba e na vegetação adjacente a uma grande indústria cerâmica, com as plantas posicionadas mais próximas à fonte emissora apresentando maiores teores foliares de F (AzEVEDO et al., 1993).

Os sintomas de toxicidade de $\mathrm{F}$ em plantas sensíveis podem ser induzidos por extensiva exposição a concentrações de $\mathrm{F}$ atmosférico de $0,6 \mathrm{mg} \cdot \mathrm{m}^{-3}(0,8$ ppb HF v/v) ou menos (COMPton e Remmert, 1960). AMUNDSON et al. (1982) encontraram sintomas típicos de toxicidade em plantas de milho doce expostas a 1,5 mg.m ${ }^{-3}$ de HF. BREWER et al. (1965) ressaltaram que sintomas podem aparecer no milho quando os teores foliares de F estão na faixa de 29 a $48 \mathrm{mg} \cdot \mathrm{kg}^{-1}$, comprometendo o rendimento da cultura acima desses valores.

Clorose foliar tem sido considerada o primeiro sintoma visual de injúria de $\mathrm{F}$ em plantas (Amundson et al., 1982; McNulty e Newman, 1961; WANDER e MCBRIDE, 1956). Concomitantemente ao aparecimento de sintomas visuais ocorre a desintegração dos cloropastos seguida pelo colapso das células (AdAms e Soldberg, 1956). A inibição da fotossíntese em milho é causada tanto pelo efeito direto do $\mathrm{F}$ no mecanismo fotossintético como pelo efeito indireto através do fechamento dos estômatos (YAMAUCHI et al., 1983). O F pode afetar os primeiros estágios da síntese de pigmentos e também induzir a degradação da estrutura do clorospasto (McNulty e Newman, 1961).

No Centro de Ciências Agrárias da Universidade Federal de São Carlos, campus de Araras, SP (CCA/UFSCar), notou-se em um dos seus ensaios de milho a presença de folhas com descolorações no limbo foliar que evoluíram até secamento precoce. Descartadas as hipóteses de deficiências nutricionais e/ ou doenças, chegou-se à suspeita de fitotoxicidade por F.

O presente trabalho objetivou avaliar os teores foliares de $\mathrm{F}$ na cultura do milho com sintomas visuais da sua toxicidade, em duas áreas próximas à possível fonte poluidora, bem como diferenças entre as cultivares de milho quanto à intensidade desses sintomas.

\section{Material e Métodos}

Neste estudo, consideraram-se duas épocas de semeadura: a de verão 2000/01 e a "safrinha" 2001, cujos experimentos foram semeados no CCA/UFSCar (22 $18^{\prime} 56^{\prime \prime} \mathrm{S}, 47^{\circ} 23^{\prime} 20^{\prime \prime} \mathrm{W}, 650 \mathrm{~m}$ de altitude) em 17/ $11 / 00$ e $23 / 3 / 01$, respectivamente, em Latossolo Vermelho Distroférrico típico, textura argilosa. Empregaram-se $350 \mathrm{~kg} \cdot \mathrm{ha}^{-1}$ da fórmula 8:28:16 + Zn $\left(\mathrm{N}, \mathrm{P}_{2} \mathrm{O}_{5}, \mathrm{~K}_{2} \mathrm{O}\right)$ no verão e $200 \mathrm{~kg} \cdot \mathrm{ha}^{-1}$ da mesma fórmula na "safrinha".

Na safra de verão, o delineamento experimental foi o de blocos ao acaso com 22 cultivares e quatro repetições. As parcelas experimentais foram constituídas por quatro linhas de 5,0 m de comprimento cada uma, empregando-se as duas centrais para a avaliação da produtividade de grãos. Aos 97 dias após a semeadura, realizou-se a avaliação visual dos sintomas, atribuindo-se notas relativas ao grau de injúria das folhas em cada parcela, conforme Bustamante (1988) para sintomas de toxicidade por F em cultivares de soja em casa de vegetação, sendo: $0=$ planta sem sintoma; 1 = sintomas iniciais de clorose; $2=\sin$ tomas moderados de clorose e $3=$ sintomas severos e necrose. 
Na "safrinha" 2001, utilizaram-se as cultivares CATI AL30, IAC-V3 e o híbrido triplo Exceler em duas áreas, localizadas em diferentes posições geográficas em relação à fonte emissora: uma a $350 \mathrm{~m}$ e outra a $1.000 \mathrm{~m}$. Foram utilizadas duas áreas para caracterizar a fitotoxicidade nas mesmas cultivares em diferentes posições geográficas, porém ao longo de uma mesma topossequência e igual classe de solo.

Aos 82 dias após a semeadura, amostras foliares foram compostas colhendo-se 40 folhas opostas às espigas de plantas localizadas em quatro pontos dispersos em $30 \mathrm{~m}$ de raio. Análises de teores de macro e micronutrientes foram feitas no Laboratório de Química e Fertilidade de Solos do DRNPA/CCA/UFSCar e, para o $\mathrm{F}$ e \% de matéria seca, no Laboratório de $\mathrm{Nu}$ trição Animal do CENA/ESALQ/USP.

Segundo o método de Frankenberger et al. (1996), o F foi extraído com ácido nítrico concentrado e determinado pelo método potenciométrico com eletrodo seletivo para F. Ressalta-se que as folhas foram previamente lavadas e congeladas para realização das análises de $\mathrm{F}$. O teste estatístico empregado para as

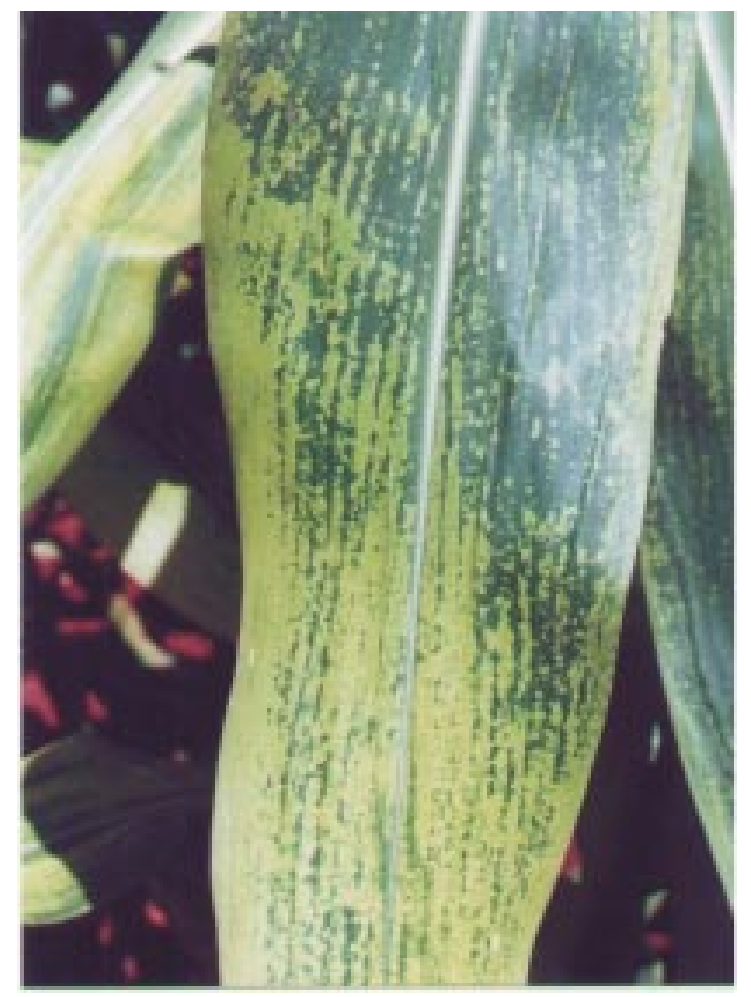

Figura 1. Sintomas iniciais de fitotoxicidade causada por fluoretos atmosféricos em folhas de milho no estádio de florescimento pleno. notas de sintomas foi o c2 de Friedman (Qui-quadrado de Friedman), o qual se adapta melhor a dados categóricos que não seguem uma distribuição normal, como é o caso das notas de sintomas.

\section{Resultados e Discussão}

Na safra de verão, observaram-se sintomas pouco antes do florescimento, iniciando por clorose internerval nas folhas mais expostas ao sol e evoluindo para necrose marginal das folhas no período da maturação dos grãos (Figuras 1 e 2). A intensidade dos sintomas foi maior no estádio de enchimento de grãos, nas duas épocas de semeadura.

Houve efeito significativo das cultivares em produtividade e em notas de sintomas ao nível de $5 \%$ (Quadro 1). Em soja, Bustamante (1988) encontrou diferença entre cultivares na sensibilidade à toxicidade de F. No presente trabalho, constatou-se que as cultivares Savana 185 e Graúna 183 apresentaram maiores notas do que os genótipos AG 1051, BRS 2110, BRS 2114 e C 333B.

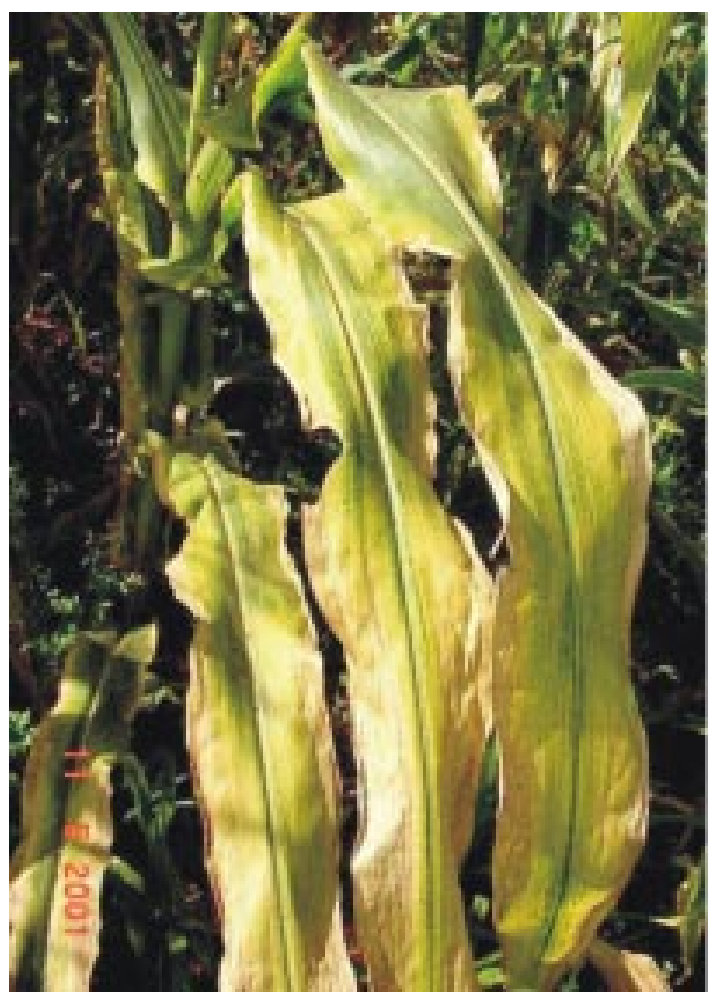

Figura 2. Clorose e necrose marginais em folhas de milho, no estádio de enchimento de grãos. 
Quadro 1. Produtividade de cultivares de milho em Araras (SP), na safra de verão 2000/01, notas de sintomas de fitotoxicidade de $\mathrm{F}$ aos 97 dias após a semeadura e parâmetros de teste Qui-quadrado de Friedman para notas

\begin{tabular}{lrr}
\hline \multirow{2}{*}{ Cultivares } & Produtividade & Notas de Sintoma \\
\cline { 2 - 3 } & Sodiana & Soma dostos $\left(\mathrm{R}_{\mathrm{i}}\right)$
\end{tabular}

AG 1051

8.123 a

1,0

20,0

C 333B

$7.834 \mathrm{ab}$

1,0

20,0

CATI AL 30

$7.249 \mathrm{abc}$

2,0

48,0

AG122

$7.230 \mathrm{abc}$

1,0

28,5

XB 8010

$7.102 \mathrm{abc}$

2,0

48,0

AL Bandeirantes

$6.963 \mathrm{abc}$

1,5

38,5

AGN 2012

$6.902 \mathrm{abc}$

1,5

38,0

Graúna 133

$6.804 \mathrm{abc}$

2,0

66,5

Balú 184

$6.507 \mathrm{abc}$

2,5

71,0

BR 2010

$6.476 \mathrm{abc}$

1,0

38,0

AL 34

$6.364 \mathrm{abc}$

1,5

38,5

IAC Airã

$6.357 \mathrm{abc}$

1,5

38,0

AL 25

$6.159 \mathrm{abc}$

2,0

58,0

SHS 4040

$6.125 \mathrm{abc}$

2,0

48,0

BRS 2114

$6.095 \mathrm{abc}$

1,0

20,0

BRS 2110

$6.086 \mathrm{abc}$

1,0

20,0

Traktor

$6.030 \mathrm{abc}$

2,0

65,5

Graúna 183

$5.985 a b c$

3,0

79,5

AL Manduri

$5.901 \mathrm{bc}$

1,0

29,5

Savana 185

$5.788 \mathrm{bc}$

3,0

84,0

AGN 3100

$5.405 \mathrm{c}$

2,0

58,0

IAC Vitória

$5.128 \mathrm{c}$

2,0

56,5

DMS Tukey 5\%

2.211

DMS $c^{2}$ Friedman 5\%

66

C.V. (\%) 12,76

8,89

* Médias seguidas de mesma letra não diferem no teste Tukey a 5\%. 
Quadro 2. Resultados das análises químicas de folhas de cultivares de milho "safrinha" amostradas nas áreas distantes 350 e 1.000 m da indústria cerâmica, em Araras (SP), em 2001

\begin{tabular}{|c|c|c|c|c|c|c|c|c|c|c|c|c|}
\hline Cultivar & $\mathrm{N}$ & $\mathrm{P}$ & $\mathrm{K}$ & $\mathrm{Ca}$ & $\mathrm{Mg}$ & S & B & $\mathrm{Cu}$ & $\mathrm{Fe}$ & $\mathrm{Mn}$ & $\mathrm{Zn}$ & F \\
\hline
\end{tabular}

$350 \mathrm{~m}$

\begin{tabular}{|c|c|c|c|c|c|c|c|c|c|c|c|c|}
\hline Exceler & 13,5 & 1,1 & 15,4 & 4,0 & 1,8 & 1,6 & 19 & 10 & 442 & 50 & 24 & 187,1 \\
\hline CATI AL30 & 19,0 & 1,1 & 17,7 & 3,8 & 1,8 & 1,4 & 11 & 8 & 240 & 47 & 36 & 162,2 \\
\hline IAC V3 & 24,0 & 1,3 & 16,6 & 4,0 & 2,0 & 1,9 & 9 & 9 & 432 & 57 & 25 & 129,3 \\
\hline & \multicolumn{12}{|c|}{$1.000 \mathrm{~m}$} \\
\hline Exceler & 24,0 & 1,2 & 15,8 & 5,2 & 1,8 & 1,8 & 5 & 7 & 205 & 40 & 30 & 116,5 \\
\hline CATI AL30 & 21,5 & 1,3 & 16,2 & 3,3 & 2,1 & 1,8 & 8 & 7 & 200 & 51 & 24 & 126,6 \\
\hline IAC V3 & 21,5 & 1,1 & 16,6 & 3,4 & 2,1 & 2,0 & 11 & 8 & 222 & 51 & 25 & 136,2 \\
\hline
\end{tabular}


Os sintomas foliares indicam a toxicidade de $\mathrm{F}$, mas sua intensidade não evidencia a magnitude que os processos fisiológicos da planta são afetados. Segundo JACOBSON et al. (1966), a grande variabilidade entre espécies e cultivares de plantas quanto à suscetibilidade ao F pode ser explicada pelas diferenças tanto em acumulação quanto em translocação e distribuição do F. Em milho, RATHORE (1992) observou redução de área foliar, taxa de assimilação liquida e taxa de crescimento.

Embora a maioria das cultivares com notas maiores tenha sido menos produtiva, não se pode atribuir este fato diretamente à fitotoxicidade de $\mathrm{F}$, devido à natural interação genótipo-ambiente. Por exemplo, as cultivares Balu 184 e CATI 183 figuraram no grupo das mais produtivas mesmo apresentando notas elevadas de sintomas (Quadro 1).

Os teores foliares de nutrientes nas duas áreas de milho "safrinha" estavam dentro da faixa de suficiência (Quadro 2). Nesse ciclo observaram-se sintomas de toxicidade nas folhas do milho somente depois do início do florescimento, mas devido à baixa intensidade, não foram avaliados por notas. Não foi possível diferenciar esses cultivares quanto à suscetibilidade ao $\mathrm{F}$ a partir dos teores foliares. Embora a suscetibilidade das plantas ao $\mathrm{F}$ esteja associada a sua acumulação nos tecidos foliares, as diferenças não explicam a ampla faixa de sensibilidade entre espécies e cultivares de plantas. Por exemplo, gladíolos altamente sensíveis podem tornarse necróticos com $20 \mathrm{mg} \cdot \mathrm{kg}^{-1}$ de F (20 mg F/g de massa seca), enquanto plantas de algodão podem ser notadas doentes com mais de $4.000 \mathrm{mg} \cdot \mathrm{kg}^{-1}$ de $\mathrm{F}$ (JACOBSON et al., 1966).

Os valores médios de $\mathrm{F}$ nas áreas a 350 e 1.000 m da cerâmica foram de 159,5 mg. $\mathrm{kg}^{-1}$ e $126,4 \mathrm{mg} \cdot \mathrm{kg}^{-1}$ respectivamente. Esses teores são cerca de quatro vezes acima do normal aceitável para o milho, com base em BREWER et al.(1965) antes citado, o que sugere que houve a contaminação das cultivares de milho por fluoretos provenientes da indústria cerâmica.

Ressalte-se que o tempo de exposição pode determinar maior concentração de F (BUSTAMANTE, 1988). No caso desses ensaios, as amostras foram tiradas tardiamente, após a época do florescimento, enfatizando-se, assim, o caráter acumulativo do $\mathrm{F}$ nas plantas. Embora a cerâmica tenha funcionado de maneira contínua, exceto paradas mensais para manutenção dos fornos, durante este trabalho não foram avaliados os níveis de $\mathrm{F}$ no ar e suas possíveis interações com outros poluentes. Há necessidade de mais estudos para se correlacionar notas de diagnose visual de sintomas com teores de F nos tecidos e possíveis prejuízos na produtividade.

\section{Conclusão}

Foi possível constatar elevados teores de $\mathrm{F}$ em folhas de milho com sintomas visuais da sua toxicidade. Constatou-se variabilidade genética entre as cultivares de milho quanto à intensidade dos sintomas observados.

\section{Agradecimentos}

À pesquisadora científica Gisèle M. Fantin, do Instituto Biológico, em Campinas (SP), pela foto (Figura 1) e tentativas iniciais de diagnóstico; ao Eng. ${ }^{\circ}$ Agr. ${ }^{\circ}$ Vítor J. Cicolin, da Associação Agroindustrial de Cascalho, pelo material bibliográfico e ao Grupo de Estudo e Pesquisa em Agricultura, GEPAGRI/ CCA/UFSCar, pelo auxílio na realização dos experimentos.

\section{Referências Bibliográficas}

ADAMS, D.F.; SOLBERG, R.A. Histological responses of some plant leaves to hydrogen fluoride \& sulfur dioxide. American Journal of Botany, Columbus, v.43, p. 755-760, 1956.

AMUNDSON, R.G.; WEINSTEIN, L.H.; VAN LEUKEN, P.; COLAVITO, L.J. Joint action of HF and NO2 on growth, fluorine accumulation, and leaf resistance in marcross sweet corn. Environmental and Experimental Botany, Oxford, v.22, p.49$55,1982$.

ARNDT, U.; FLORES, F.E.V.; WEINSTEIN, L.H. Efeito do F sobre as plantas: diagnose de danos na vegetação do Brasil. Porto Alegre: Universidade Federal do Rio Grande do Sul, 1995. 155p.

ASSOCIAÇÃO AGROINDUSTRIAL DE CASCALHO. Contaminação de F nas lavouras do Bairro do Cascalho. Cordeirópolis, mar., 2001. 54p. (Relatório Técnico).

AZEVEDO, C.M.A.; DINIZ, K.M.; PRADELLA, D.Z.A. Contaminação da vegetação por fluoretos em Cordeirópolis, SP. São Paulo: CETESB, 1993. 12p. (Informação Técnica).

BREWER, R.F.; GUILLEMET, F.B.; SHUTHERLAND, F.H. The effects of atmospheric fluoride on gladiolus growth, flowering and corn production. Proceedings of American Society for Horticultural Science, Alexandria, v.88. p.634-644, 1965.

BUSTAMANTE, M.M.C. Efeitos fisiológicos e bioquímicos do F em dois cultivares de soja (Glycine max L. (Merril.)). 1988. 76f. Dissertação (Mestrado) - Universidade Federal de Viçosa.

COMPTON, O.C.; REMMERT, L.F. Effect of air-borne fluorine on injury and fluorine content of gladiolus leaves. Proceedings of American Society for Horticultural Science, Alexandria, v.75, p.663-675, 1960. 
FIALHO, R.C. Acumulação foliar de fluoretos e seu significado ecológico em espécies arbóreas da Mata Atlântica, Cubatão, SP. 1997. 100f. Dissertação (Mestrado) - Instituto de Biociências, Universidade de São Paulo, São Paulo.

FIGUEIREDO, J.G. Avaliação de gramíneas tropicais como bioindicadoras da presença do F. 1994. 89f. Dissertação (Mestrado) - Universidade Federal de Viçosa.

FRANKENBERGER, W.T.; TABATABAI, M.A.; ADRIANO, D.C.; DONER, H.E. Bromine, Chlorine and Fluorine. In: Methods of Soil Analysis: Chemical Methods. Part 3. Madison, Wisconsin,USA: SSSA, 1996. p.847-867.

JACOBSON, J.S.; WEINSTEN, L.H.; McCUNE, D.C.; HITCHCOCK, A.E. The accumulation of fluorine by plants. Journal of Air Pollution Control Association, Pittsburgh, v.16, p.412417, 1966.
LOPES, M.R.; MACHADO, S.P.; SOUZA, J.B. Revisão da metodologia de monitoramento ativo de fluoreto com o bioindicador Cordyline terminalis. São Paulo: CETESB, dez., 1998. 42p. (Relatório Técnico).

MCNULTY,I.B.; NEWMAN, D.W. Mechanisms of fluoride induced chlorosis. Plant Physiology, Sofia, v.36, p.385-388, 1961.

RATHORE, S. Effect of fluoride toxicity on leaf area, net assimilation rate and relative growth rate of Hordeum vulgare and Zea mays. Fluoride, Warren, v.25, p.175-183, 1992.

WANDER, I.W.; McBRIDE Jr., J.J. Chlorosis produced by fluorine on citrus in Florida. Science, Washington, v.123, p.933-934, 1956.

YAMAUCHI, M.; CHOI, W.K.; YAMADA, Y. Fluoride Inhibition of photosynthesis in certain crop plants. Soil Science and Plant Nutrition, Tokyo, v.29, p.549-553, 1983. 